\title{
Prevalence of Intestinal Parasitic Infections and Associated Factors Among Diarrheal Outpatients in South Gondar Zone, Northwest, Ethiopia
}

Getaneh Yemata ( $\nabla$ gechat9@gmail.com )

DebreTabor University ,College of Health Sciences, Social and Public health Unit

\section{Melkalem Azanaw}

DebreTabor University ,College of Health Sciences, Social and Public health Unit

Lemma Bilal

DebreTabor University ,College of Health Sciences, Medical Laboratory Department

Birku Mekonnen

DebreTabor University ,College of Health Sciences, Medical Laboratory Department

\section{Research Article}

Keywords: confidence interval, NMHC, Ethiopia

Posted Date: November 30th, 2020

DOI: https://doi.org/10.21203/rs.3.rs-109505/v1

License: (9) This work is licensed under a Creative Commons Attribution 4.0 International License.

Read Full License 
Prevalence of Intestinal parasitic infections and associated factors among diarrheal outpatients in South Gondar Zone, Northwest, Ethiopia.

Getaneh Atikilt Yemata $^{1 *}$, MelkalemMamuyeAzanaw ${ }^{1}, \quad$ BirkuGashaw Mekonnen $^{2}$
,LemmaWorkine Bilal ${ }^{2}$

\section{Affiliations}

${ }^{1}$ Department of Social and PopulationHealth, College Health Sciences, Debre Tabor University, Debre Tabor, Ethiopia

${ }^{2}$ Department of Medical Laboratory, College of Health Sciences, Debre Tabor University, Debre Tabor, Ethiopia.

Corresponding author address ${ }^{*}$

Getaneh Atikilt

Email: gechat9@gmail.com 


\begin{abstract}
Background: Intestinal parasitic infections are major public health problems in several tropical and subtropical developing countries with poor socio-economic status including Ethiopia. Diarrheal patients are a major part of the population with a high risk of intestinal parasitosis. There is a scarcity of studieson intestinal parasite infection among diarrheal patients in South Gondar. As a result, this study was conducted to assess the prevalence and associated factors of intestinal parasite infection among diarrheal patients in this area.
\end{abstract}

Methods: An institutional-based cross-sectional study design was conducted from March to May 2019 with a structured questionnairedata collection technique. A total sample of 240diarrheal patients included in the study and a simple random sampling technique was used. The stool sample from each study subject was collected and analyzed by direct wet mount techniques. Data were entered in Epi Data Version 4.6.0.0 and exported to Stata version 14 for analysis. Binary logistic regression was used to measure the association of factors with the outcome variable. Factors that have an association with the outcome variable at p-value 0.25 was taken into the multivariable logistic regression analysis to control the potential confounders. The result of the final model will be expressed in terms of adjusted Odd Ratios (AOR) and 95\% confidence interval (CI) and statistical significance will be declared if the P-value is less than 0.05.

\title{
Results:
}

The overall prevalence of intestinal parasitic infections among 240 diarrheal patients was $45.4 \%$. The majorities of the positive cases were single infections (85.2\%) while the remains were double infections (14.8\%). Among the intestinal parasitic infections Entamoeba histolytica/dispar (E. histolytica/dispar) was the highest 60 (55.5\%) and followed by Giardia lamblia (G. lamblia) 26 (24.1\%), Ascaris lumbricoides (A. lumbricoides) 4(3.7\%), hookworm4(3.7\%) and Enterobius vermicularis 4(3.7\%). The presence of intestinal parasitic infections had significantly associated with marital status (AOR: 0.41; 95\% CI: 0.18,0.91).),shoe-wearing habit(AOR:2.81; 95\% CI:1.07,7.37) and fingernail cleanliness (AOR:5.12; 95\% CI: 2.35,11.12).

\section{Conclusion:}

Intestinal parasitic infections were a highly prevalent health problem among populations in Lay Gaynte woreda.Poor hygiene of fingernails and no shoes wearing a habit were risk factors for intestinal parasites infection among diarrheal patients 


\section{Introduction}

Intestinal parasitic infections affected more than 3.5 billion people globally and leads to clinical morbidity for around 450 million individuals $(1,2)$.

Intestinal Parasites (IP) are organisms that infect the gastrointestinal tract of humans and other animals. Helminths and protozoans are the main types of intestinal parasites. Helminths are worms with many cells, which can be divided into Nematodes (roundworms), Cestodes (tapeworms), and trematodes (flukes) while protozoan parasites are parasites that possess only one cell and can multiply inside the human body (3).

From intestinal parasitic infections; Entamoeba histolytica, Giardia lamblia, Ascaris lumbricoides (roundworm), Trichuris trichiura (whipworm), and Necator American's (hookworm) are considered to be a global public health concern and the major public health significant parasitic infections. These intestinal parasitic infections have been recognized as significant causes of illnesses and diseases worldwide (4-6).

Intestinal parasitic infections are frequently reported in the poor communities of developing countries and within these, the majority of the cases occur among diarrheal patients (7). Diarrheal diseases are the major causes of morbidity and mortality in the developing world (8). Intestinal parasitosis is more prevalent among diarrheal patients as compared with the general population. About $12 \%$ of the global disease burdens caused by intestinal parasites are observed among diarrheal patients in developing countries (9).

Intestinal parasitic infections are usually highly prevalent among the resource-poor and socioeconomically deprived communities where overcrowding, poor environmental sanitation, low level of education, and lack of access to safe water are prevalent (10). The infected people experience a vicious cycle of undernutrition and repeated infections leading to excess morbidity with children being the worst affected (9).

Health promotion and education aimed at improving personal hygiene, and community-based mass deworming are currently practiced prevention and control strategies targeted to void transmission of IP(11). However, the burden and consequence of intestinal parasitic infections are still increasing in poor communities. Regarding Giardiasis, it is the most predominant protozoan infection that affects approximately 200 million people in the world and 20 to $30 \%$ of developing countries. Amoebiasis due to infections with the intestinal protozoon Entamoeba histolytica results in 40,000-100,000 deaths each year(12,13).

Scholars claimed that environmental, social, and geographical factors such as low household income, poor personal and environmental sanitation, overcrowding, limited access to clean water, 
tropical climate, and low altitude significantly associated with the occurrence of high intestinal parasitic infections especially in tropical and sub-tropical areas $(14,15)$.

In Africa, more specifically Sub-Saharan Africa including Ethiopia, parasitic infections are highly abundant (16). For instance, recent estimates indicated that approximately 1472, 1298 and 1049 million people have roundworm, hookworm, and whipworm infection, respectively (17).

In Ethiopia, intestinal parasitic infection is one of the top ten causes of morbidity amongst children $(18,19)$. Parasitic helminths are the second most predominant causes of outpatient morbidity in the country andNorthwestern Ethiopia is highly affected as compared to other parts of Ethiopia. $(20,21)$.

Intestinal parasites particularly intestinal protozoa parasites are the major cause of diarrhea and are easily transmitted from one individual to the other through different routes including eating unwashed vegetables, contaminated food, and water. On the other way, diarrheal patients are reported to be at high risk for severe infections and mortality associated with intestinal parasitic disease(22).There is no published data in the study area that shows the prevalence of intestinal parasites among diarrheal patients and possible predictors. Therefore, the main purpose of this study was to assess the prevalence of intestinal parasites among diarrheal patients and associated risk factors.

\section{Methods and Materials}

\section{Study Area}

The study was conducted onNefas Mewecha Health Center (NMHC), South Gondar zone, Amhara regional state of northern Ethiopia. It is located $175 \mathrm{~km}$ from Bahir Dar and $739 \mathrm{~km}$ from Addis Ababa. Based on this the total population is 81,644 , of this 38,682 are males, 42,962 are females. It contains 04 kebeles. Around this kebeleswithin the town,there are one governmental andtwo private colleges, one public hospital, one public health center, four public health posts, and three private clinics. The climate is Dega and WeynaDega. NMHC is one of the health institutions in the Amhara region state that provides health services for over 10,000 inhabitants in Lay Gaynt woreda. The Health center registers and provides service for all diarrheal patients that come to this area.

Study design and study period

Across sectional study was conducted in NMHC at Nefas Mewcha (NM) town from March to May 2019.

\section{Population}

Source population

All diarrheal patients who attended NMHC during the study period 
Study population

Selected diarrheal outpatients who are attending NMHC during the study period.

\section{Eligibility Criteria}

\section{Inclusion Criteria}

Diarrheal patients that coming to NMHC and give a stool sample

\section{Exclusion Criteria}

Patients that give an inappropriate stool sample, patients who take an antihelminth drug

Sample size and sampling technique

Sample size determination

According to the study conducted in Bereka medical center in the bale zone, the sample size was determined by using the single population proportion formula. Taking (16.6\%) prevalence of IPs from a previous study (23). 95\% confidence level $(\mathrm{Z}=1.96)$ and $5 \%$ marginal error $(\mathrm{d}=0.05)$ was consideredto calculate the minimum sample size required for the study.The initial sample size was

$\mathrm{n}=\frac{(Z a / 2)^{2} * \mathrm{P} *(1-\mathrm{P})}{d^{2}}$

Where:

$\mathrm{n}=$ minimum sample size required for the study,

$Z=1.96$ (confidence interval),

$\mathrm{P}=$ proportion of the problem $(0.166)$,

$1-\mathrm{P}=1-0.166=0.834$

$\mathrm{d}=$ margin error $(5 \%) ; 95 \%$ confidence interval

$\mathrm{n}=(1.96)^{2} \times 0.166 \times 0.834=0.5318464704 / 0.0025=212.74=213$

$0.05^{2}$

$10 \%$ non-response rate $=21$, so the final sample sizewas $=244$

\section{Sampling technique}

The sampling technique was by lottery method of simple random sampling techniqueamong diarrheal outpatients that attend in health facilities of the south Gondar zone.

\section{Variables}

\section{Dependent variable}

Presence of any intestinal parasitosis (Yes/No)

Independent variables 
Age, Sex, Religion, Residence, Occupational status, education status, Availability of latrine, Water source, Habit of wearing shoes, Habit of finger nail-biting, Habit of water purification, Habit of eating raw vegetable, Habit of eating soil, Habit of handwashing and Habit of eating raw meat.

\section{Data Collection technique}

Socio-demographic characteristics and other associated factors (personal hygiene, age, sex, source of water) data were collected through face to face interview by using a pre-tested structured questionnaire. The study subject was asked to provide at least 3 grams of stool. Each stool sample was examined by using the wet mount and formal-ether concentration. The wet mount method was performed on a 0.5-gram stool and for the formal-ether concentration,the method was used 2 grams of stool the ova, cyst, and larvae of different intestinal parasites separately reported.

\section{Data Quality control}

Data quality was ensured at various activities of the study by the following standard operating procedure (SOP). The questionnaire was translated to the Amharic language by the principal investigator and at the end translated back to English. After the data collection process, the data were

checked for completeness. Before starting the actual work, the quality of the reagent and instruments were checked for the expiry date. The specimen was also being checked for the serial number, amount, consistency, quality, and procedures of collection. Positive quality control and negative quality control slides, as well as a colored atlas, were used as a guideline. Then the result of the laboratory examination was recorded in a well-prepared format carefully and finally attached with the questionnaire accordingly to their codes.

\section{Data processing and Analysis}

Data were entered in Epi Data version 4.6.0.0 and exported to STATA software version 14 for data cleaning and analysis (20). Descriptive statistics like frequency and percentage was calculated to describe the study population characteristics. The strength of associations will be measured by binary logistic regression at a $95 \%$ confidence interval and a p-value of 0.05 was being used as a cut of a point. Variables with a p-value of less than 0.2 were incorporated into multivariable logistic regression. Results were reported as AOR (adjusted odds ratio) with 95\% CI.

\section{Results}

\section{Socio-demographic characteristics among diarrhea patients}

A total of 240diarrheal patients were included forthe final investigation. However, 4(1.6\%) were excluded because of the inability to provide specimens. Among the study participants, 131 
$(54.6 \%)$ patients were female. Nearly halves $(48.7 \%)$ of the study participants were from a rural place of residence and 84 (35.0\%) had no formal education i.e. unable to read or write. A higher proportion of respondents were married (50.4\%), Orthodox Christian (83.8\%), and Farmer $(44.6 \%)($ Table1).

Table 1: Socio-demographic characteristics of diarrheal patients who are attending in health facilities of the South Gondar zone, Northwest Ethiopia from March-May 2019 (N=240).

\begin{tabular}{|c|c|c|c|}
\hline Variables & Category & Frequency & Percent \\
\hline \multirow[t]{2}{*}{ Sex } & Male & 109 & 45.4 \\
\hline & Female & 131 & 54.6 \\
\hline \multirow[t]{2}{*}{ Residence } & Urban & 123 & 51.3 \\
\hline & Rural & 117 & 48.7 \\
\hline \multirow[t]{3}{*}{ Age in years } & Less than Fifteen & 62 & 25.8 \\
\hline & Between 15 to 49 & 156 & 65.0 \\
\hline & Greater than 49 & 22 & 9.2 \\
\hline \multirow[t]{2}{*}{ Religion } & Orthodox & 201 & 83.8 \\
\hline & Muslim & 39 & 16.2 \\
\hline \multirow[t]{3}{*}{ Marital Status } & Single & 99 & 41.3 \\
\hline & Married & 121 & 50.4 \\
\hline & Divorced/ Widowed & 20 & 8.3 \\
\hline \multirow[t]{3}{*}{ Educational Status } & No education & 84 & 35.0 \\
\hline & Primary education & 82 & 34.2 \\
\hline & $\begin{array}{l}\text { Secondary education and } \\
\text { above }\end{array}$ & 74 & 30.8 \\
\hline \multirow{5}{*}{$\begin{array}{l}\text { Occupational } \\
\text { Status }\end{array}$} & Government employee & 23 & 9.6 \\
\hline & Farmer & 107 & 44.6 \\
\hline & Merchant & 37 & 15.4 \\
\hline & Student & 61 & 25.4 \\
\hline & Other & 12 & 5.0 \\
\hline Total & & 240 & $100 \%$ \\
\hline
\end{tabular}

\section{Hygiene and Sanitation related characteristics}


Almost all (90.0\%) of the study participants came from a household that had a latrine facility. Of the total participants, about $45.4 \%$ had a habit of handwashing after the toilet and $86.6 \%$ had a habit of wearing shoes. Moreover, halves $(52.9 \%)$ of the study participants used tap water sources.Most (80\%) of the study participants had practicing fingernail trimming (Table 2).

Table 2: Hygiene and sanitation-related characteristics of diarrheal patients who are attending in health facilities of South Gondar zone, Northwest Ethiopia from March-May 2019 (N=240).

\begin{tabular}{|c|c|c|c|}
\hline Variables & Category & Frequency & Percent \\
\hline \multirow[t]{2}{*}{ Latrine availability } & No & 24 & 10.0 \\
\hline & Yes & 216 & 90.0 \\
\hline \multirow[t]{2}{*}{ A habit of handwashing after toilet } & No & 131 & 54.6 \\
\hline & Yes & 109 & 45.4 \\
\hline \multirow[t]{4}{*}{ Water source } & Tap & 127 & 52.9 \\
\hline & Well & 16 & 6.6 \\
\hline & Stream & 75 & 31.3 \\
\hline & River & 22 & 9.2 \\
\hline \multirow[t]{2}{*}{ A habit of shoe-wearing } & No & 32 & 13.4 \\
\hline & Yes & 207 & 86.6 \\
\hline \multirow[t]{2}{*}{ A habit of water purification } & No & 220 & 91.7 \\
\hline & Yes & 20 & 8.3 \\
\hline \multirow[t]{2}{*}{ A habit of eating raw meat } & No & 152 & 63.3 \\
\hline & Yes & 88 & 36.7 \\
\hline \multirow[t]{2}{*}{ Is fingernail trimmed? } & No & 48 & 20.0 \\
\hline & Yes & 192 & 80.0 \\
\hline \multirow[t]{2}{*}{ A habit of soil eating } & No & 235 & 97.9 \\
\hline & Yes & 5 & 2.1 \\
\hline \multirow[t]{2}{*}{ A habit of eating raw vegetable } & No & 132 & 55.0 \\
\hline & Yes & 108 & 45.0 \\
\hline Total & & 240 & $100 \%$ \\
\hline
\end{tabular}

\section{Prevalence of intestinal parasites and distribution of parasites}

The overall prevalence of single or multiple intestinal parasitic infections among diarrheal patients was $45.4 \%$ with a $95 \%$ CI of $39.2 \%$ to $51.8 \%$. Different types of parasites were detected from the stool samples of study participants. Prevalence of Entamoeba histolytica/dispar (E. histolytica/dispar) was the highest $60(55.5 \%)$ and followed by Giardia lamblia (G. lamblia) 
26(24.1\%), Ascaris lumbricoides (A. lumbricoides) 4(3.7\%), hookworm4(3.7\%)and Enterovirus vermicularis $4(3.7 \%)$. The majorities of the positive cases were single infections $(85.2 \%)$ while the remains were double infections (14.8\%) (Figure 1).

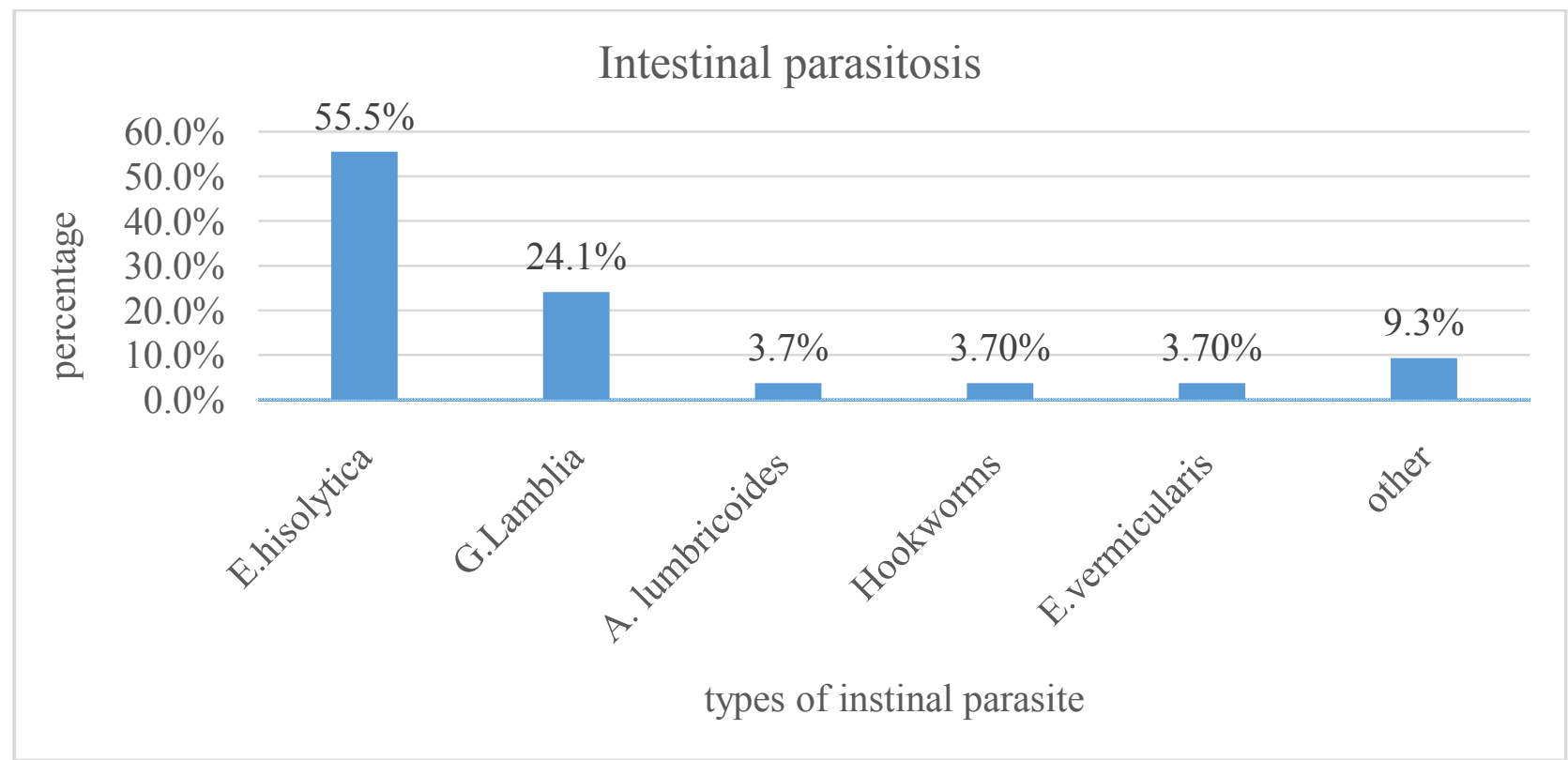

Figure 1:Prevalence species of intestinal parasites among diarrheal patients for ova, trophozoite, and oocysts of parasites who attending health facilities in south Gondar zone, Northwest Ethiopia, 2019.

\section{Factors associated with intestinal parasites among diarrheal patients}

Sex, age, religion, marital status, a habit of handwashing after toilet, a habit of purifying water before drinking, shoe-wearing habit, and cleanliness of finger was incorporated into multivariable logistic regressionsince P-value less than 0.25. According to multiple logistic regressionshoewearing habit,marital status, and cleanliness of fingerswere statistically significant associated factorsto intestinal parasitic infections (Table 3).

The odds of having intestinal parasites among married diarrheal patients were lower by $51 \%$ compared to single marital status (AOR: 0.41; 95\% CI: 0.18,0.91). Besides, the likelihood of occurring intestinal parasites among patients who did not wear shoes frequently was 2.81 times higher compared to those who wear frequently (AOR:2.81; 95\% CI:1.07,7.37). Furthermore, patients who had no clean fingernails 5.12times higher to acquirethe intestinal parasitic infection as compared to those who had clean fingernails(AOR:5.12;95\% CI: 2.35,11.12) (Table 3). 
Table 3: Factors associated with intestinal parasites among diarrheal patients who were attending in health facilities of South Gondar zone, Northwest Ethiopia,2019.

\begin{tabular}{|c|c|c|c|}
\hline Factors & Category & COR with 95\% CI & AOR with $95 \%$ CI \\
\hline \multirow[t]{2}{*}{ Sex } & Male & 1 & 1 \\
\hline & Female & $0.60(0.36,1.00)$ & $0.98(0.51,1.86)$ \\
\hline \multirow[t]{2}{*}{ Religion } & Orthodox Christian & 1 & 1 \\
\hline & Muslim & $0.48(0.23,0.99)$ & $0.95(0.41,2.19)$ \\
\hline \multirow[t]{3}{*}{ Age in years } & Less than 15 & 1 & 1 \\
\hline & $15-49$ & $0.48(0.26,0.87)$ & $0.81(0.34,1.89)$ \\
\hline & Greater than 49 & $0.72(0.27,1.92)$ & $1.14(0.29,4.41)$ \\
\hline \multirow[t]{3}{*}{ Marital status } & Single & 1 & 1 \\
\hline & Married & $0.49(0.29,0.85)$ & $0.41 *(0.18,0.91)$ \\
\hline & Divorced/widowed & $0.833(0.32,2.18)$ & $1.30(0.41,4.14)$ \\
\hline \multirow{2}{*}{$\begin{array}{l}\text { A habit of handwash- } \\
\text { ing after toilet }\end{array}$} & Yes & 1 & 1 \\
\hline & No & $1.56(0.93,2.60)$ & $1.17(0.65,2.10)$ \\
\hline \multirow{2}{*}{$\begin{array}{l}\text { A habit of shoe- } \\
\text { wearing }\end{array}$} & Yes & 1 & 1 \\
\hline & No & $3.03(1.37,6.73)$ & $2.81 *(1.07,7.37)$ \\
\hline \multirow[t]{2}{*}{ Use purified water } & Yes & 1 & 1 \\
\hline & No & $3.65(1.18,11.27)$ & $2.56(0.76,8.54)$ \\
\hline \multirow[t]{2}{*}{ Fingers status } & Trimmed & 1 & 1 \\
\hline & Not trimmed & $4.89(2.39,10.00)$ & $5.12 * * *(2.35,11.12)$ \\
\hline
\end{tabular}

\section{DISCUSSION}

This study assessed the prevalence of different intestinal parasitic infections and associated risk factors inNMHC. From the results obtained, the prevalence of intestinal parasites was analyzed by sex; the males had a higher frequency of $52.8 \%$ of IPIs, whereas the females had a lower frequency rate of $47.2 \%$ of IPIs. It was inline with different local and international studies $(20,21,24)$.

The overall prevalence of intestinal parasites infection among patients in NMHC was $45.4 \%$ (95\% CI:(39.2\% -51.8\%)). It was in line with astudy conducted among multicenter in Northern Ethiopia (36). Besides this the findings of our study were higher as compared to astudy con- 
ducted in Brazile, India, and other local studies; Arbaminch,Mekelle, and Addis Ababa(25-28). However, it was lower as compared to findings in Yemen and studies in the Amhara region;Motta health centers, and districts in Bahirdar(25,29).

In this study, the most prevalent parasitic infection was Entamoebahistolytica $(55.5 \%)$ followed by Giardia lamblia (24.1\%). Ascaris lumbricoid,Hookworm, and Enterobius vermicularis was the least in prevalence.The pattern and distribution of IPs were similar to astudy conducted in Yemen, Arba Minch(25,26).

Those patients who had no clean fingernailshad 5.12 times higherodds to havean intestinal parasitic infection as compared to those who had clean fingernails.Similar to this finding, a study conducted in different setups showed that fingernail status is significantly associated with intestinal parasitic infection $(20,25,26)$. The association could be justified as the leading IPs in this study Enteameoba histolytica and Giardia lamblia primarily transmittedfecal-orally.Unclean fingernails havethe main roleinthe fecal-oral transmission of intestinal parasites through not washed or unclean hands.

The odds of intestinal parasitic infections were 2.81 times higher among the diarrheal patients who had no shoe wearing habit as compared to those who wear shoes frequently. Similar to this study conducted in Debreelias and Bahir Dar showed that patients who had no habit of wearing shoes were more infected with intestinal parasites than havinga habit of wearing shoes $(19,29)$. This could be explained through; $3.7 \%$ of diarrheal patients who visited the targeted health centers were infected with hookworm parasites. Being barefooted or not wearing shoes is the main route of exposure for hookworm. This finding implies that some parts of the community practiced shoe-wearing less frequently and a proportion of them was infected with hookworm and other pathogens.

Age, sex, a habit of handwashing after toilet, and usage of purified water were not statistically associated with the occurrence of intestinal parasitic infections in this study.

\section{Conclusion}

Intestinal parasitic infections were a highly prevalent health problem among populations in Lay Gaynteworeda. The risk factor of the prevalence of intestinal parasites was poor hygiene of fingernails and no shoes wearing a habit.All these factors including shoe-wearing habits and keeping fingernails clean play an important role in preventing patients from infection with intestinal parasites. The habit of wearing shoes was found to be a protective factor for hookworm infection.

Therefore, the local District Health Office and other governmental and non-governmental organizations need to give attention to this serious problem of an intestinal parasitic infection of the 
patients. The health professional should be provided health information with special emphasis on the importance of personal hygiene and the habit of wearing shoes in case of intestinal parasitic infection.

\section{Abbreviations and acronyms:}

CI: Confidence interval, DTU: Debre Tabor University,HC: Health center, IPIs:Intestinal Parasitic Infections, MLS: Medical laboratory science, WHO: World Health Organization,NMHC: Nefas Mewcha Health Center.

\section{Declarations}

\section{Ethical Consideration}

The study was approved by Debre Tabor University, college of health science ethical review committee. Permission was obtained fromthe administrator and workers of NMHC. The health center authorities, professionals, and the patients were informed about the purpose and procedures of the study. Informed consent from each adult respondent and informed assent from legal guardians or parents of children were obtained.Confidentiality was observed and an unauthorized person is not access to the data collected. Each subject was assigned a study identification number, and these subject identifiers will notbe released outside the researcher. Data is only accessed by the authorized person. The methods and procedures were conducted according to Debretabor university regulations and guidelines.

\section{Consent for publication}

Not applicable

Availability of data and materialsThe data sets in this study are available from the corresponding author on reasonable request

Competing interestsThe authors declare no competing interests

FundingNo fund was obtained for this particular study

Authors' contributionsG.A. and M.M. wrote the manuscript including tables and figures.B.G. and L.W. have contributed to methods. The final manuscript is read and approved by all authors

\section{Acknowledgments}

The authors would like to acknowledge Debre Tabor University, College of Health Science for approving the ethical review process. The authors are also wanted to express gratitude to data collectors, NMHC staff, supervisors, and study participants.

\section{REFERENCE}

1. Tigabu A, Taye S, Aynalem M, Adane K. Prevalence and associated factors of intestinal parasitic infections among patients attending Shahura Health Center, Northwest Ethiopia. BMC Res Notes 
[Internet]. BioMed Central; 2019;1-8. Available from: https://doi.org/10.1186/s13104-019-4377-y

2. Pullan RL, Smith JL, Jasrasaria R, Brooker SJ. Global numbers of infection and disease burden of soil transmitted helminth infections in 2010. 2014;1-19.

3. L. S. STEPHENSON MCLEAO. Malnutrition and parasitic helminth infections. 2020;(Table 2).

4. Ngui R, Ishak S, Chuen CS, Mahmud R, Lim YAL. Prevalence and Risk Factors of Intestinal Parasitism in Rural and Remote West Malaysia. 2011;5(3):1-7.

5. Oyegue-liabagui SL, Ndjangangoye NK, Kouna LC, Lekolo GM, Mounioko F, Nolna SK, et al. Molecular prevalence of intestinal parasites infections in children with diarrhea in Franceville , Southeast of Gabon. BMC Infectious Diseases; 2020;1-11.

6. Bahk YY, Shin E, Cho S, Ju J, Chai J, Kim T. Prevention and Control Strategies for Parasitic Infections in the Korea Centers for Disease Control and Prevention. 2018;56(5):401-8.

7. WHO intestinal parasitosis. Report of the WHO Informal Consultation on the Use of Chemotherapy for the Control of Morbidity Due to Soil- Transmitted Nematodes in Humans , Geneva , 29 April to 1 May 1996 Files in this item. 2020;1-3.

8. World Health Organization. Diarrhoeal disease. 2017;2-5.

9. Al-mekhlafi HM, Al-maktari MT, Jani R, Ahmed A, Anuar TS, Moktar N, et al. Burden of Giardia duodenalis Infection and Its Adverse Effects on Growth of Schoolchildren in Rural Malaysia. 2013;7(10):1-12.

10. Saidin S, Othman N, Noordin R. Update on laboratory diagnosis of amoebiasis. European Journal of Clinical Microbiology \& Infectious Diseases; 2019;15-38.

11. MUIRURI-GITAHI MGW. Effects of public health interventions on intestinal parasitic infections among school-going children in Muranga country Kenya. 2016;(June).

12. Ghazzay, Muna Hashim et al. Prevalence of amoebic dysentery and giardiasis in Al-Najaf. Int J Res Pharm Sci. 2019;(10.4):2960-2964.

13. Seguí R, Muñoz-antoli C, Klisiowicz DR, Oishi CY, Köster PC, Lucio A De, et al. Prevalence of intestinal parasites, with emphasis on the molecular epidemiology of Giardia duodenalis and Blastocystis sp ., in the Paranaguá Bay , Brazil : a community survey. Parasites \& Vectors; 2018;1-19.

14. Wegayehu T, Tsalla T, Seifu B, Teklu T. Prevalence of intestinal parasitic infections among highland and lowland dwellers in Gamo area, South Ethiopia. 2013;

15. WHO G. Prevention and control of intestinal parasitic infections. 1987;

16. Hall A, Hewitt G, Tuffrey V, Silva N De. Review Article A review and meta-analysis of the impact of intestinal worms on child growth and nutrition. 2008;118-236.

17. Bentwich Z, Kalinkovich A. Immune activation is a dominant factor in the pathogenesis of African AIDS. 1995;16.

18. Mulatu G, Zeynudin A, Zemene E, Debalke S, Beyene G. Intestinal parasitic infections among children under five years of age presenting with diarrhoeal diseases to two public health facilities in Hawassa , South Ethiopia. Infect Dis Poverty [Internet]. Infectious Diseases of Poverty; 
2015;1-8. Available from: http://dx.doi.org/10.1186/s40249-015-0081-x

19. Hailegebriel T. Prevalence of intestinal parasitic infections and associated risk factors among students at Dona Berber primary school, Bahir Dar ,. BMC Infectious Diseases; 2017;1-8.

20. Abossie A, Seid M. Assessment of the prevalence of intestinal parasitosis and associated risk factors among primary school children in Chencha town ,. 2014;1-8.

21. Mengistu A, Gebre-selassie S, Kassa T. Prevalence of intestinal parasitic infections among urban dwellers in southwest Ethiopia. (16):12-7.

22. Azam A, Peerzada MN, Ahmad K. Parasitic diarrheal disease : drug development and targets. $2015 ; 6: 1-12$.

23. S Taye AA et. a. Prevalence of Intestinal Parasitic Infections among Patients with Diarrhea at Bereka Medical Center, Southeast Ethiopia. Med Sci Res. 2014;

24. Ghazzay MH, Kiad SJ, Shamran SJ, Abdulbary M, Plant M. Prevalence of amoebic dysentery and giardiasis in Al-Najaf Muna. Int J Res Pharm Sci. 2019;10(4):2960-4.

25. Alwabr GMA, Al-moayed EE. Prevalence of intestinal parasitic infections among school children of Al-Mahweet Governorate, Yemen. 2016;6(2):64-73.

26. Mama M, Alemu G. Prevalence and factors associated with intestinal parasitic infections among food handlers of Southern Ethiopia: cross sectional study. BMC Public Health [Internet]. BMC Public Health; 2016;1-7. Available from: http://dx.doi.org/10.1186/s12889-016-2790-x

27. Megbaru Alemu et al. Epidemiology of Intestinal Parasite Infections among Kindergarten Children in. Int J Pharma Sci Res. 2015;(November).

28. Beyene G, Tasew H. Prevalence of intestinal parasite, Shigella and Salmonella species among diarrheal children in Jimma health center, Jimma southwest Ethiopia: a cross sectional study. $2014 ; 1-7$.

29. Publishing E, Asemahagn MA, Sciences H. Parasitic Infection and Associated Factors among the Primary School Children in Motta Town, Western. 2014;2(6):248-54. 
Figures

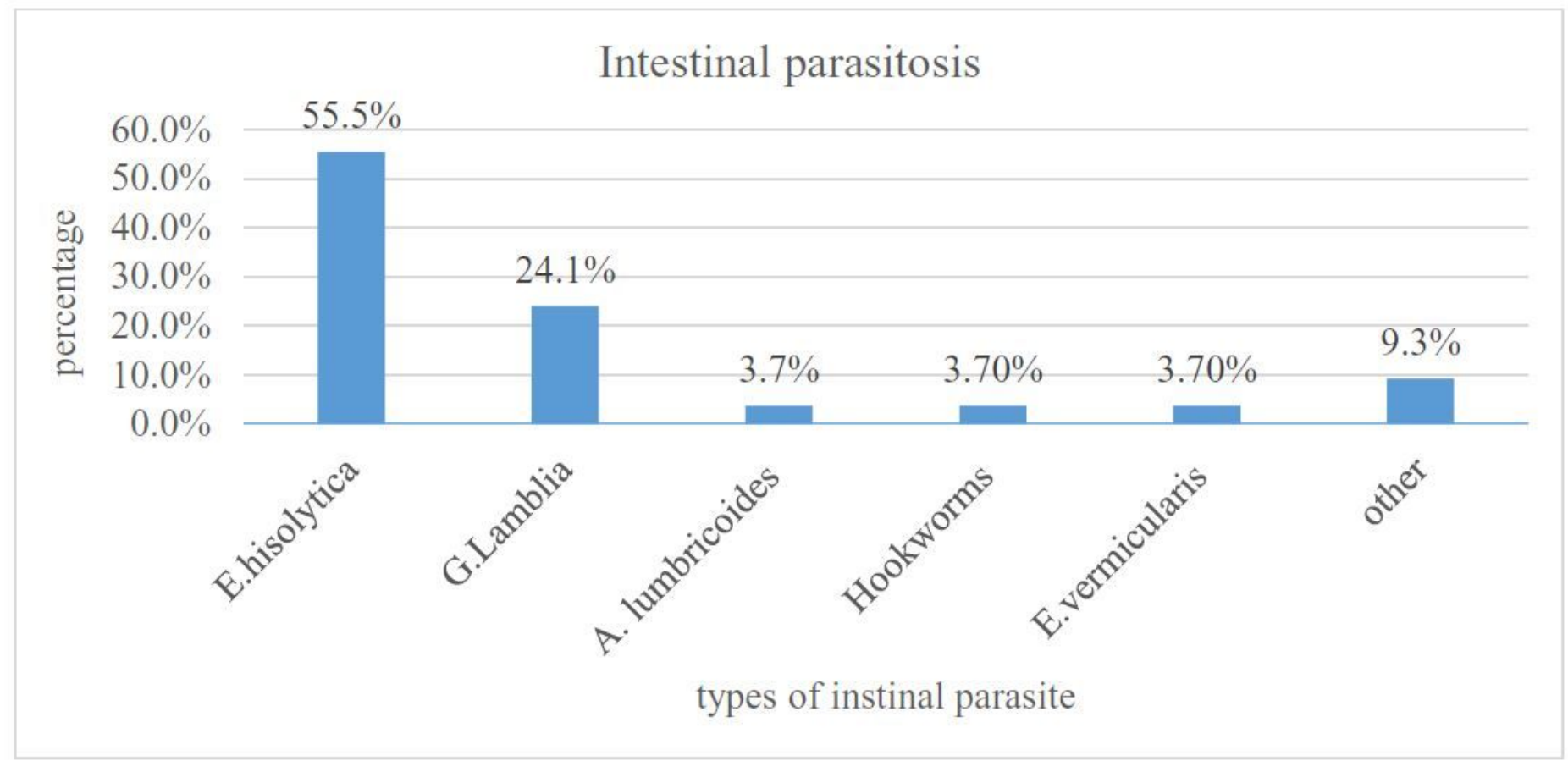

\section{Figure 1}

Prevalence species of intestinal parasites among diarrheal patients for ova, trophozoite, and oocysts of parasites who attending health facilities in south Gondar zone, Northwest Ethiopia, 2019. 